\title{
A Pattern Classification Based Approach for Blur Classification
}

\author{
Shamik Tiwari \\ CET, Mody University of Science \& Technology, Lakshmangarh \\ e-mail: shamiktiwari@hotmail.com
}

\begin{abstract}
Blur type identification is one of the most crucial steps of image restoration. In case of blind restoration of such images, it is generally assumed that the blur type is known prior to restoration of such images. However, it is not practical in real applications. So, blur type identification is extremely desirable before application of blind restoration technique to restore a blurred image. An approach to categorize blur in three classes namely motion, defocus, and combined blur is presented in this paper. Curvelet transform based energy features are utilized as features of blur patterns and a neural network is designed for classification. The simulation results show preciseness of proposed approach.
\end{abstract}

Keywords: Blur, Motion, Defocus, Curvelet Transform, Neural Network.

\section{Introduction}

Barcoding is one of the automatic identification and data collection (AIDC) technology which reduces human involvement in data entry and collection and in that way also dropping error and time in the process. These days, a wide range of general purpose hand-held devices, such as mobile phones, come with an optical imaging system. Enabling these general purpose hand-held devices with the capability to recognize barcodes is a cost-effective alternative to conventional barcode scanners $[1,2]$. The availability of imaging phones provides people a mobile platform for decoding barcode rather than the use of the conventional scanner which has lack of mobility.

Unfortunately, sometimes the deprived quality of the images taken by digital cameras makes it difficult to correctly decode barcodes. Barcode images are usually prone to number of degradations. Image blurring is often a major factor influencing the performance of a barcode recognition system. Image blurring is usually inevitable in a camera-based imaging system, especially in the case of the camera that does not have auto focus or macro mode $[3,4]$. Although some high-end camera phones which integrate a high resolution and auto focus/macro mode are available, the low-end camera phone user segment is still huge.

The objective of image restoration approach is to recover a true image from a degraded version. This problem can be stated as blind or non-blind depending upon whether blur parameters are known prior to the restoration process. Blind restoration deals with parameter identification before deconvolution. Though there exists multiple blind restorations techniques but blur type recognition is extremely desirable before application of any blur parameters estimation approach [5].

While most previous work focuses on image deblurring, not as much research has been done on blur classification, which is more practical because the type of blurs is usually unknown in images. Based on the descriptor of blurs, there are a few blur classification methods. Multilayer neural network is a neural network, which is based on the multi valued neurons (MLMVN) with traditional feed forward architecture. The MLMVN is used by Aizenberg et al. [6] to identify types of the blur, whose precise identification is essential for the image deblurring. Chong et al. [7] have proposed a scheme that simultaneously detects and identifies blur. This method is based on the analysis of extrema values in an image. The extrema of histograms are first identified then analyzed in order to extract feature values in this method. Liu et al. [8] have offered a framework for partial blur detection and classification i.e. whether some portion of the image is blurred as well as what type of blur arises. They considered maximum saturation of color, gradient histogram span and spectrum details as blur features. Aizenberg et al. [9] presented a work which identifies blur type, estimates blur parameters and perform image 
restoration using neural network. They considered four kinds of blur namely rectangular, motion, defocus, and Gaussian blurs as a pattern classification problem. Yan et al. [10] made an attempt to find a general feature extractor for common blur kernels with various parameters, which is closer to realistic application scenarios and applied deep belief neural networks for discriminative learning. In this method Fourier spectrum of blurred images is passed to the neural network as input. However, utilizing spectrum as an input is not a good idea due to large size of feature set. In the paper [11], Tiwari et al. use statistical features of blur patterns in frequency domain and identified blur type with the use of feed forward neural network. Tiwari et al. [12] used wavelet features for blur type recognition utilizing a neural network.

Curvelet features have been extensively used in object and texture classification. However, we have not found any application of curvelet features for blur classification. In this paper we extract curvelet features of blur patterns in frequency domain and use feed forward neural network for blur classification. This paper is organized into seven sections including the present section. In section two to four, we discuss the theory of image degradation model, curvelet transform and feed forward neural network in that order. Section five describes the methodology of blur classification scheme. Section six discusses experimental results and in the final section seven, conclusion is presented.

\section{Image Degradation Model}

The image degradation process of an image can be modelled as the following convolution process [12].

$$
g(x, y)=f(x, y) \otimes h(x, y)+\eta(x, y)
$$

where $g(x, y)$ is the degraded image, $f(x, y)$ is the uncorrupted original image, $h(x, y)$ is the point spread function that caused the degradation and $\eta(x, y)$ is the additive noise in spatial domain. In view of the fact that, convolution operations used in spatial domain is equivalent to the multiplication in frequency domain, so image degradation model is

$$
G(u, v)=F(u, v) H(u, v)+N(u, v)
$$

Motion blur occurs in an image due to relative motion between image capturing device and the object. Let the image to be acquired has a relative motion to the capturing device by a regular velocity (vrelative) and makes an angle of a radians with the horizontal axis for the duration of the exposure interval [0, texposure], the distortion is one dimensional. Expressing motion length as $L=v_{\text {relative }} \times t_{\text {exposure, }}$, the point spread function (PSF) for uniform motion blur can be modeled as [12]:

$$
h(x, y)= \begin{cases}\frac{1}{L} \text { if } \sqrt{x^{2}+y^{2}} \leq \frac{L}{2} \text { and } \frac{x}{y}=-\tan \alpha \\ 0 \quad \text { otherwise }\end{cases}
$$

The defocus blur also known as out of focus blur is appears due to a system of circular aperture. It can be modeled as a uniform disk as [13]:

$$
h(x, y)= \begin{cases}\frac{1}{\pi R^{2}} & \text { if } \sqrt{x^{2}+y^{2}} \leq R \\ 0 & \text { otherwise }\end{cases}
$$

where $\mathrm{R}$ defines the radius of the disk.

Sometimes image contains co-existence of both blurs. In that case the blur model becomes [14]:

$$
h(x, y)=a(x, y) \otimes b(x, y)
$$

where $\mathrm{a}(\mathrm{x}, \mathrm{y}), \mathrm{b}(\mathrm{x}, \mathrm{y})$ are point spread functions for motion and defocus blur respectively and $\otimes$ is the convolution operator. The degradation process model equations (1) and (2) can be expressed as (6) and (7) in spatial and frequency domain respectively: 


$$
\begin{aligned}
& g(x, y)=f(x, y) \otimes a(x, y) \otimes b(x, y)+\eta(x, y) \\
& G(u, v)=F(u, v) A(u, v) B(u, v)+N(u, v)
\end{aligned}
$$

\section{Discrete Curvelet Transform}

Curvelet Transform by Candes and Donoho $[15,16]$ was designed to overcome the inherent limitations of traditional multiscale transforms like wavelet. Curvelet transform is a multi-scale and multi-directional transform with needle shaped basis functions. Basis functions of wavelet transform are isotropic and thus it requires large number of coefficients to represent the curve singularities. Curvelet transform basis functions are needle shaped and have high directional sensitivity and anisotropy. Curvelets obey parabolic scaling.The curvelet has a frequency support in a parabolic wedge area due to the anisotropic scaling law as width $=$ length2.

The fast discrete curvelet transform was introduced by Candes et al. [17] in two forms, the wrapping version and the unequally spaced FFT (USFFT) version. Since the wrapping version is faster and invertible up to numerical precision, while the USFFT version is only approximately invertible, we use only the wrapping version throughout this paper. The two dimensional curvelet transform is given by

$$
C^{D}(j, l, k)=\sum_{x=0}^{M-1} \sum_{y=0}^{N-1} f[x, y] \varnothing_{j, l, k}^{D}[x, y]
$$

where $f[x, y], 0 \leq x<M, 0 \leq y<N$ is the input 2-D image. $C^{D}(j, l, k)$ are the discrete curvelet coefficients, $\emptyset_{\mathrm{j}, 1, \mathrm{~L}}^{\mathrm{D}}[\mathrm{x}, \mathrm{y}]$ is the curvelet basis function at scale $\mathrm{j}$, orientation $\mathrm{l} \in[0,2 \pi]$ and location $\mathrm{k}\left[\mathrm{k}_{1}, \mathrm{k}_{2}\right]$.

\section{Feed Forward Neural Network}

A successful pattern identification method depends significantly on the particular selection of the classifier. An artificial neural network (ANN) is a system which can be seen as an information-processing paradigm. ANN has been designed as generalizations of mathematical models identical to human recognition system. They are collection of interconnected processing units called neurons that perform as a unit. It can be used to determine complex relationships among inputs and outputs by recognizing patterns in data. The feed forward neural network refers to the neural network which contains a set of source nodes which forms the input layer, one or more than one hidden layers, and single output layer. In case of feed forward neural network input signals propagate in one direction only; from input to output. There is no feedback path i.e. the output of one layer does not influence same layer. One of the best known and broadly acceptable learning algorithms in training of multilayer feed forward neural networks is Back-Propagation [18]. The back propagation is a type of supervised learning algorithm, which means that it receives sample of the inputs and associated outputs to train the network, and then the error (difference between real and expected results) is calculated [19]. The idea of the back propagation algorithm is to minimize this error, until the neural network learns the training data. This can be implemented by:

$$
\Delta w_{j i}(n+1)=\eta\left(\partial_{p j} O_{p j}\right)+\alpha \Delta w_{j i}(n)
$$

where $\eta_{\text {is }}$ the learning rate, $\alpha$ is the momentum, ${ }^{\partial j}$ is the error and $\mathrm{n}$ is the number of iteration.

\section{Blur Classification Approach}

Blur type can be identified in frequency domain notably. The motion blur, defocus blur are appeared differently in frequency domain, and the blur categorization can be easily done using these specific patterns. The frequency spectrum of motion blurred image has dominant

IJEEI Vol. 5, No. 2, June 2017: $162-173$ 
dark parallel lines, which are orthogonal to the motion orientation with near zero values. On the other hand, In case of defocus blur one can see appearance of some circular zero crossing patterns and in case of coexistence of both blurs combined effect of both blurs become visible. Figure 1 shows the effect of different blurs on the Fourier spectrum of original image. We can consider these patterns in frequency domain as image itself for blur classification.

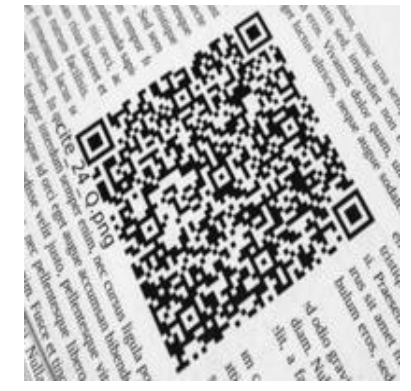

(a)

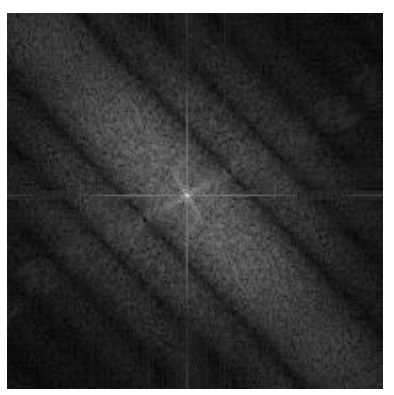

(d)

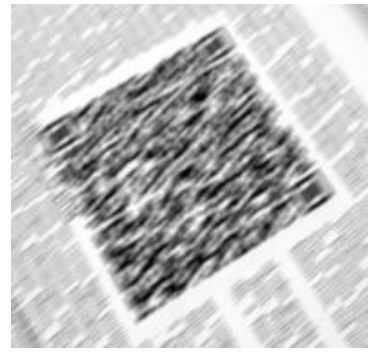

(b)

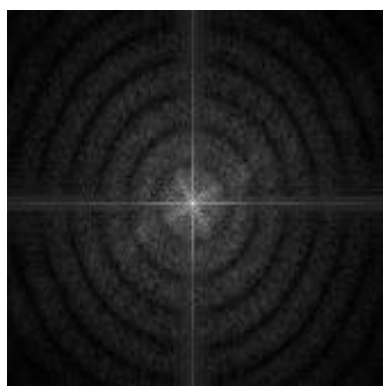

(e)

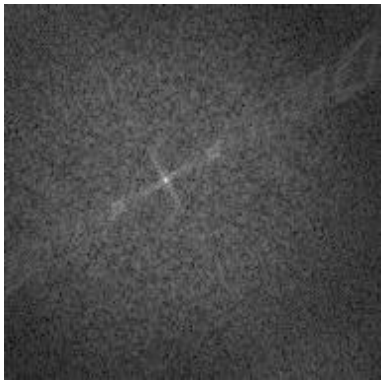

(c)

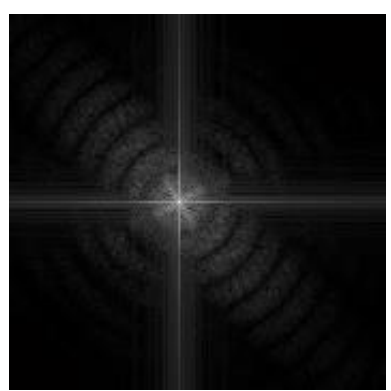

(f)

Figure 1(a) Original image containing QR code [18] (b) Motion blurred image

(c) Fourier spectrum of original image (d) Fourier spectrum of blurred image with motion length 10 pixels and motion orientation 450 (e) Fourier spectrum of blurred image with defocus blur of radius 5 and (f) Fourier spectrum of image with coexistence of both blurs

Main steps of the blur classification method are: image preprocessing, find logarithmic frequency spectrum, feature extraction of blur patterns, designing of neural network classifier system and result analysis.

In the initial step, we perform preprocessing on blurred images. Then we find the logarithmic frequency spectrum of blurred and non blurred image to get the blur patterns. Once the blur patterns acquired, the curvelet energy features have been calculated to prepare the training and testing database. Finally with this feature database training and testing are performed by using feed forward back propagation neural network.

\subsection{Preprocessing}

Blur classification requires a number of preprocessing steps. First, the color image obtained by the digital camera is changed into an 8-bit grayscale image by averaging the color channels.

The periodic transitions at the boundaries of an image often lead to high frequencies, which are transformed into visible vertical and horizontal lines in the power spectrum. Since, these lines may divert from or even superpose the stripes caused by the blur, they have to be detached by applying a windowing function before transformation. The Hanning window gives a fine trade-off between forming a smooth transition towards the image borders and keeping 
adequate image information in power spectrum. A 2-D Hann window of size $\mathrm{N} \mathrm{X} \mathrm{M}$ defined as the product of two 1-D Hann windows:

$$
w[n, m]=\frac{1}{2}\left(1+\cos \left[2 \pi \frac{n}{N}\right]\right) \cdot \frac{1}{2}\left(1+\cos \left[2 \pi \frac{m}{M}\right]\right)
$$

After that step, the windowed image is transformed to the frequency domain using Fourier transform. Then, the power spectrum is achieved. However, as the coefficients of the spectrum decrease rapidly from its centre to the borders, it is difficult to identify local differences significantly. This fast drop off is reduced by considering the logarithm of the power spectrum. In order to obtain a centered version of the spectrum, its quadrants have to be swapped diagonally. A centered portion of size $256 \times 256$ is cropped to perform feature extraction, in view of the fact that the significant features are around the centre of the spectrum,

\subsection{Feature Extraction}

Wrapping based discrete curvelet transform using Curvelab-2.1.2 is applied to a power spectrum of barcode image to obtain its coefficients. These coefficients are then used to form the features of blur patterns. After achieving the curvelet coefficients the mean and standard deviation of the coefficients related with each subband is calculated at the coarsest and the finest scale independently. The mean of a subband at scale $\mathrm{j}$ and orientation lis calculated as:

$$
\mu_{\mathrm{j} . \mathrm{l}}=\frac{\mathrm{E}(\mathrm{j}, \mathrm{l})}{\mathrm{M} \times \mathrm{N}}
$$

where $\mathrm{M} \times \mathrm{N}$ is the size and $\mathrm{E}(\mathrm{j}, \mathrm{l})$ is the energy of curvelet transformed image respectively at scale $\mathrm{j}$ and orientation $\mathrm{l}$. Energy is calculated by the sum of absolute values of curvelet coefficients.

$$
E(j, l)=\sum_{x=0}^{M-1} \sum_{y=0}^{N-1}\left|C_{j, l}^{D}(x, y)\right|
$$

The standard deviation of a subband at can be shown as:

$$
\sigma_{\mathrm{j}, \mathrm{l}}=\frac{\sqrt{\sum_{\mathrm{x}=0}^{\mathrm{M}-1} \sum_{\mathrm{y}=0}^{\mathrm{N}=1}\left(\left|\mathrm{c}_{\mathrm{j}, 1}^{\mathrm{D}}(\mathrm{x}, \mathrm{y})\right|-\mu_{\mathrm{j}, \mathrm{I}}\right)^{2}}}{\mathrm{MXN}}
$$

We have used 3 level discrete curvelet decomposition. Table 1 shows the subbands division at each level of transform.

Table 1. Curvelet transform with 3 levels

\begin{tabular}{|l|ccc|} 
Scale & 1 & 2 & 3 \\
Total No. of subbands & 1 & 16 & 32
\end{tabular}

Only first half of the total subbands at a scale are considered for feature calculation because in the frequency domain the curvelet at angle l produces the similar coefficients as the curvelet at angle $(1+\pi)$. Based on the subband division in table $1,(1+8+16)=25$ subbands of curvelet coefficients are selected for calculation of mean and standard deviation. All these features are arranged in a manner such that the standard deviations remain in the first half of the feature vector and the means are arranged into the second half of the feature vector. So, we obtain a feature vector consists of mean energies and standard deviations $f=\left(f_{1}, f_{2} \ldots, f_{50}\right)^{T}$ for each of the power spectrum. Where first twenty five values are standard deviation and others are mean energies. We use this feature vector $f$ to classify blur patterns

IJEEI Vol. 5, No. 2, June 2017 : $162-173$ 


\section{Simulation Results}

The performance of the proposed technique has been evaluated using camera based barcode images. To simulate the blur classification algorithm, we have considered two different barcode image databases. The first database WWU Muenster Barcode Database [20] consisting of 1-D barcode images and the second one is the Brno Institute of Technology QR code image database [21] captured by digital camera. This complete work is implemented using neural network and image processing toolbox of Matlab 6.5.

Most of pattern recognition problems are too complex to be solved completely by non automated algorithms, machine learning has always played a crucial role in this area. The conventional way of identification is to split the recognizer into a feature extractor, and a classifier. One of the main achievements of the neural network is to offer complex mapping in high-dimensional spaces without requiring complicated hand-crafted features. This allows designers to rely more on learning, and less on detailed engineering of feature extractors. These features of neural network enforced to use as a classification tool in this work.

The classification is achieved using a feed forward neural network containing single hidden layer fifty neurons. Back propagation is pertained as network training principle, where the training dataset is designed by the extracted features of the blur patterns. The whole training and testing features set is normalized into the range of $[0,1]$, whereas the output class is assigned to zero for the lowest probability and one for the highest. In this work the transfer functions of hidden layers are hyperbolic tangent sigmoid functions. The numbers of neurons used in input layer are equal to the extracted features from image dataset which are fifty. The neural classifier is trained with different number of hidden layers. The final architecture is selected with single hidden layer of fifty neurons which gives best performance.

To design the neural network, the data is divided into training, validation and test sets in a ratio of 50:20:30 respectively. Neural networks are known to be prone to overfitting, and hence not performing well on previously-unseen data. In order to reduce the risk of overfitting, a validation dataset was used to test each network periodically during training. When the validation set error began to rise, training was terminated. Only at that point the network was tested with the test data set in order to calculate the final accuracy. Three different partition sets were created using random partition. Partitions satisfied cross-validation, meaning that each target class (i.e. blur) was represented the same number of times in the training set, the same number of times in the validation set and the same number of times in the test set. For each partition, every pattern appeared in exactly one of the training, validation or test sets. Finally, we used overall classification results to evaluate the blur classification model.

When referring to the performance of a classification model, we are interested in the model's ability to correctly predict or separate the classes. When looking at the errors made by a classification model, the confusion matrix gives the full picture. In confusion matrix, the diagonal cells show the number of instances that were correctly classified for each structural class. The off-diagonal cells show the number of instances that were misclassified. To assess the blur classification model, we have shown confusion matrix and used the four statistical metrics as specified below.

Precision (Positive Predictive Value): Precision measures the fraction of samples that truly turns out to be positive in the group the classifier has categorized as a positive class. The higher the precision is, the lower the number of false positive errors committed by the classifier.

$$
\text { Precision }=\frac{\text { True Positive }}{\text { True Positive }+ \text { False Positive }}
$$

Sensitivity (Recall): It measures the actual members of the class which are accurately recognized as such. It is also referred as true positive rate (TPR). It is defined as the fraction of positive samples categorized accurately by the classification model. High sensitivity value represents that low number of positive samples misclassified as the negative class.

$$
\text { Recall }=\frac{\text { True Positive }}{\text { True Positive }+ \text { False Negative }}
$$


Specificity: Recall/sensitivity is related to specificity, which is a measure that is commonly used in multi class problems, where one is more interested in a particular class. Specificity corresponds to the true-negative rate.

$$
\text { Specificity }=\frac{\text { True Negative }}{\text { True Negative }+ \text { False Positive }}
$$

Accuracy: Accuracy is the overall correctness of the model and is calculated as the sum of correct classifications divided by the total number of classifications.

Where the terms true positive, true negative, false positive and false negative are defined below.

(i) True Positive: In case of test pattern is positive and it is categorized as positive, it is considered as a true positive.

(ii) True Negative: In case of test pattern is negative and it is categorized as negative, it is considered as a true negative.

(iii) False Positive: In case of test pattern is negative and it is categorized as positive, it is considered as a false positive.

(iv) False Negative: In case of test pattern is positive and it is categorized as negative, it is considered as a false negative

Experiment 1: Performance analysis with blurred 1-D images

In the first experiment, we have considered first 200 images from the 1-D barcode database. Then, the three different classes of blur (motion, defocus and combined blur) were synthetically introduced with different parameters to make the database of 600 blurred images (i.e., 200 images with each class of blur) for each type of barcode images. Out of 600 blurred images, 300, 120 and 180 images are used as training, validation and testing datasets respectively to design the neural model. The training process is continued till the best validation performance achieved. The training stopped when the validation error increased for 6 iterations. The best validation performance is 0.0115 , at epoch 39 as shown in Figure. 2

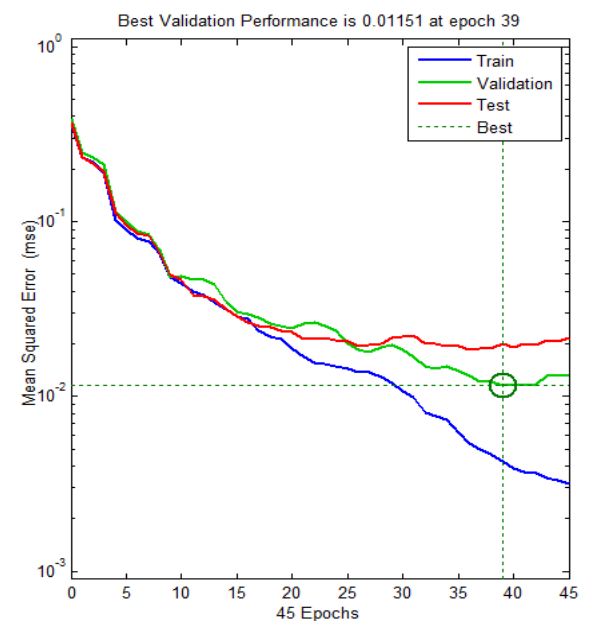

. Figure 2. Network Performance

Table 2. Confusion matrix for blur classification results of blurred 1-D barcode images

\begin{tabular}{|c|c|c|c|c|}
\hline \multicolumn{5}{|c|}{ Target Class } \\
\hline Predicted & & Motion & Defocus & Combined \\
\hline \multirow{4}{*}{ Class } & & Blur & Blur & Blur \\
\hline & Motion & 189 & 0 & 0 \\
\hline & $\begin{array}{l}\text { Defocus } \\
\text { Blur }\end{array}$ & 11 & 200 & 0 \\
\hline & $\begin{array}{l}\text { Combined } \\
\text { Blur }\end{array}$ & 0 & 0 & 200 \\
\hline
\end{tabular}

IJEEI Vol. 5, No. 2, June 2017 : 162 - 173 
Table 3. Blur Classification results for blurred 1-D barcode images

\begin{tabular}{lccc}
\hline \multicolumn{1}{c}{ Blur Type } & Precision & Recall & Specificity \\
\hline Motion Blur & 100 & 94.5 & 100 \\
Defocus Blur & 94.8 & 100 & 97.2 \\
Combined & 100 & 100 & 100 \\
Blur & Accuracy & & 98.2 \\
& & & \\
\hline
\end{tabular}

The trained network model is used subsequently to categorize the blur type from degraded images. The overall blur classification results are presented as confusion matrix in Table 2. Using this confusion matrix statistical quantities precision, recall, specificity and accuracy are calculated. The high values of these metrics in Table 3 show effectiveness of our model. The overall classification accuracy is $98.2 \%$.

Experiment 2: Performance analysis with blurred and noisy 1-D images

To test the efficiency of the proposed scheme in presence of noise, another neural model is created using 2001 -D barcode images. All the images are degraded different kinds of blur similar to Experiment 1. Subsequently, Gaussian noise of 40dB Blurred Signal-to-Noise Ratio (BSNR) is added to the blurred images to create a database of blurred and noisy samples.

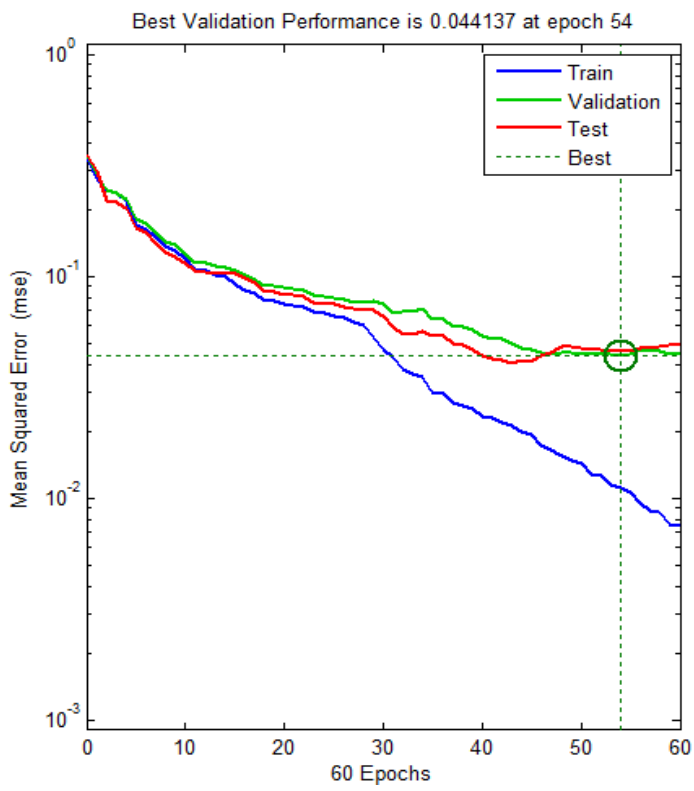

Figure 3. Network Performance

Table 4. Confusion matrix for blur classification results of blurred and noisy

\begin{tabular}{|c|c|c|c|c|}
\hline \multicolumn{5}{|c|}{ Target Class } \\
\hline \multirow[t]{4}{*}{$\begin{array}{l}\text { Predicted } \\
\text { Class }\end{array}$} & & $\begin{array}{c}\text { Motion } \\
\text { Blur }\end{array}$ & $\begin{array}{c}\text { Defocus } \\
\text { Blur }\end{array}$ & $\begin{array}{c}\text { Combined } \\
\text { Blur }\end{array}$ \\
\hline & $\begin{array}{l}\text { Motion } \\
\text { Blur }\end{array}$ & 199 & 10 & 0 \\
\hline & $\begin{array}{l}\text { Defocus } \\
\text { Blur }\end{array}$ & 1 & 183 & 17 \\
\hline & $\begin{array}{l}\text { Combined } \\
\text { Blur }\end{array}$ & 0 & 7 & 183 \\
\hline
\end{tabular}


Table 5. Blur Classification results for blurred and noisy 1-D barcode images

\begin{tabular}{cccc}
\hline Blur Type & Precision & Recall & Specificity \\
\hline Motion Blur & 95.2 & 99.5 & 97.5 \\
Defocus Blur & 91.0 & 91.5 & 99.5 \\
Combined Blur & 96.3 & 91.5 & 98.2 \\
& Accuracy & & 94.2 \\
\hline
\end{tabular}

The training process is continued till the best validation performance achieved. The training stopped when the validation error increased for 6 iterations. The best validation performance is 0.0441 , at epoch 54 as shown in Figure 3 . The trained network model is used subsequently to classify the blur type from degraded images. Table 4 shows the confusion matrix of the proposed blur classification system. Table 5 shows the performance of the proposed model in terms of precision, sensitivity, specificity, and classification accuracy. The high values of these metrics show effectiveness of our model. The overall classification accuracy is $94.2 \%$.

Experiment 3: Performance analysis with blurred 2-D images

In this experiment, we have considered first 200 images from the 2-D barcode database. Afterward, the three different classes of blur (motion, defocus and combined blur) were artificially introduced to each barcode image with varying degree of parameters to construct the database of 600 blurred images (i.e., 200 images with each type of blur). Out of these 600 blurred images, 300, 120 and 180 images are utilized as training, validation and testing datasets respectively to design the neural model. The training process is continued till the best validation performance achieved. The training stopped when the validation error increased for 6 iterations. The best validation performance is 0.0067 , at epoch 40 as shown in Figure 4. The trained network model is used subsequently to identify the blur type from degraded images. The overall blur classification results are presented as confusion matrix in Table 6. By this confusion matrix statistical quantities precision, recall, specificity and accuracy are calculated. Table 7 shows these metrics. The high values of these metrics show effectiveness of our model. The overall classification accuracy is achieved as $98.7 \%$.

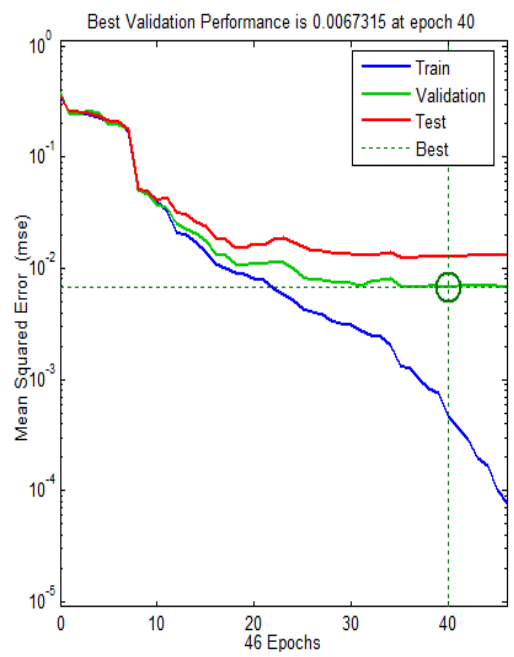

Figure 4. Network Performance

IJEEI Vol. 5, No. 2, June 2017: $162-173$ 
Table 6. Confusion matrix for blur classification results of blurred 2-D barcode images

\begin{tabular}{|c|c|c|c|c|}
\hline \multirow{5}{*}{$\begin{array}{c}\text { Predicted } \\
\text { Class }\end{array}$} & \multicolumn{3}{|c|}{ Target Class } & \multirow[b]{2}{*}{$\begin{array}{c}\text { Combined } \\
\text { Blur }\end{array}$} \\
\hline & & $\begin{array}{c}\text { Motion } \\
\text { Blur }\end{array}$ & $\begin{array}{l}\text { Defocus } \\
\text { Blur }\end{array}$ & \\
\hline & $\begin{array}{l}\text { Motion } \\
\text { Blur }\end{array}$ & 199 & 5 & 0 \\
\hline & $\begin{array}{l}\text { Defocus } \\
\text { Blur }\end{array}$ & 1 & 194 & 1 \\
\hline & $\begin{array}{c}\text { Combined } \\
\text { Blur }\end{array}$ & 0 & 1 & 199 \\
\hline
\end{tabular}

Table 7. Blur Classification results for blurred 2-D barcode images

\begin{tabular}{cccc}
\hline Blur Type & Precision & Recall & specificity \\
\hline Motion Blur & 97.5 & 99.5 & 98.7 \\
Defocus Blur & 99.0 & 97.0 & 99.5 \\
Combined Blur & 99.5 & 99.5 & 99.7 \\
& Accuracy & & 98.7 \\
\hline
\end{tabular}

Experiment 4: Performance analysis with blurred and noisy 2-D images

To examine the efficiency of the proposed method in presence of noise, another neural model is created using 200 2-D barcode images. First, all the images are degraded different kinds of blur similar to Experiment 3. Then, Gaussian noise of $40 \mathrm{~dB}$ BSNR is introduced to the blurred images to create a database of blurred noisy samples. The training process is continued till the best validation performance achieved. The training terminated when the validation error increased for 6 iterations. The best validation performance is 0.0188 , at epoch 23 as shown in Figure 5. The trained network model is used subsequently to recognize the blur type from degraded images. The performance of model is presented in Table 8 and Table 9. The overall classification accuracy is $96.3 \%$.

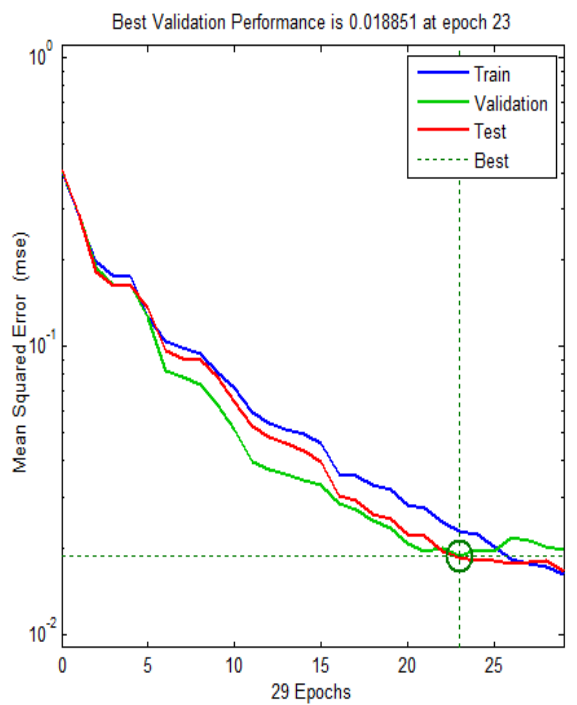

Figure 5. Network Performance

Table 8. Confusion matrix for blur classification results of blurred and noisy 2-D barcode images

\begin{tabular}{|c|c|c|c|c|}
\hline \multirow{5}{*}{$\begin{array}{c}\text { Predicted } \\
\text { Class }\end{array}$} & \multicolumn{3}{|c|}{ Target Class } & \multirow[b]{2}{*}{$\begin{array}{c}\text { Combined } \\
\text { Blur }\end{array}$} \\
\hline & & Motion & Defocus & \\
\hline & Motion Blur & 196 & 10 & 0 \\
\hline & Defocus Blur & 4 & 185 & 3 \\
\hline & Combined Blur & 0 & 5 & 197 \\
\hline
\end{tabular}


Table 9. Blur Classification results for blurred and noisy 2-D barcode images

\begin{tabular}{cccc}
\hline Blur Type & Precision & Recall & Specificity \\
\hline Motion Blur & 95.1 & 98.0 & 97.5 \\
Defocus Blur & 96.4 & 92.5 & 98.3 \\
Combined Blur & 97.5 & 98.5 & 98.7 \\
& Accuracy & & 96.3 \\
\hline
\end{tabular}

In Table 10 results of proposed method are also compared with another method which uses statistical features for blur classification. It is obvious that proposed method gives better results in comparison to this method.

Table 10. A comparison of blur classification results

\begin{tabular}{ccc}
\hline Experiment Data Sets & $\begin{array}{c}\text { Statistical features based } \\
\text { method [12] }\end{array}$ & $\begin{array}{c}\text { Proposed method using } \\
\text { curvelet energy features }\end{array}$ \\
\hline 1-D blurred images & 96.7 & 98.2 \\
1-D blurred and noisy images & 95.0 & 94.2 \\
2-D blurred images & 98.0 & 98.7 \\
2-D blurred and noisy images & 95.2 & 96.3 \\
\hline
\end{tabular}

\section{Conclusion}

In this paper, we have proposed a new blur classification scheme for barcode images taken by digital cameras. The scheme makes use of the ability of curvelet features to discriminate blur patterns appear in frequency spectrum of blurred images. This work identifies blur type, which can further help to choose the appropriate blur parameter estimation approach for blind restoration of barcode images. We have also show that in presence of low level noise, performance of blur classification system is precise. The limitation of this method is that presence of high level of noise causes disappearance of blur patterns in frequency spectrum, which cause the poor performance. Further work extension can be made to improve the performance of the classifier system with the high level of noise by incorporating the noise filtering techniques.

\section{Acknowledgment}

We highly appreciate College of Engineering and Technology, Mody University of Science \& Technology, Lakashmangarh for providing facility to carry out this research work.

\section{References}

[1] Kato H, Tan KT. Pervasive 2D barcodes for camera phone applications. IEEE Pervasive Computing. 2007; 6(4): 76-85.

[2] Yang $\mathrm{H}$, Alex $\mathrm{C}$, Jiang $\mathrm{X}$. Barization of low-quality barcode images captured by mobile phones using local window of adaptive location and size. IEEE Trans. on Image Processing. 2012; 21 (1): 418-425.

[3] Joseph E, Pavlidis T. Bar code waveform recognition using peak locations. Pattern Analysis and Machine Intelligence. IEEE Transactions on. 1994; 16(6): 630-640.

[4] Selim E. Blind deconvolution of barcode signals. Inverse Prob. 2004; 20(1): 121- 135.

[5] Tiwari S, Shukla VP, Biradar SR, Singh AK. Review of motion blur estimation techniques. J Image Graph. 2013; 1(4): 176-184.

[6] Aizenberg I, Aizenberg N, Bregin T, Butakov C, Farberov E, Merzlyakov N, and Milukova O. Blur Recognition on the Neural Network based on Multi-Valued Neurons. Journal of Image and Graphics. 2000; 5: 127-130.

[7] Chong RM, Tanaka T. Image extrema analysis and blur detection with identification. IEEE International Conference on Signal Image Technology and Internet Based Systems. 2008: 320-326.

[8] Liu R, Li Z, Jia J. Image partial blur detection and classification. In Proc. CVPR. 2008: 23-28.

[9] Aizenberg I, Paliy DV, Zurada JM, Astola JT. Blur identification by multilayer neural network based on multivalued neurons. IEEE Transactions on Neural Networks. 2008; 19(5): 883-898.

[10] Yan R, Shao L. Image Blur classification and parameter identification using two-stage deep belief networks. British Machine Vision Conference (BMVC), Bristol, UK. 2013: 1-11.

IJEEI Vol. 5, No. 2, June 2017: $162-173$ 
[11] Tiwari S, Shukla VP, Sangappa Biradar AS. Texture Features based Blur Classification in Barcode Images. 2013.

[12] Tiwari S, Shukla VP, Biradar SR, Singh AK. Blur Classification Using Wavelet Transform and Feed Forward Neural Network. International Journal of Modern Education and Computer Science (IJMECS). 2014; 6(4): 16.

[13] Tiwari S, Shukla VP, Biradar SR, Singh AK. Blind Restoration of Motion Blurred Barcode Images using Ridgelet Transform and Radial Basis Function Neural Network. Electronic Letters on Computer Vision and Image Analysis. 2014; 13(3): 63-80.

[14] Tiwari S, Shukla VP, Biradar SR, Singh AK. Defocus Blur Parameter Estimation in Barcode Images using Fast Discrete Curvelet Transform. International Journal of Tomography \& Simulation ${ }^{\mathrm{TM}}$. 2014; 27(3): 35-46.

[15] Tiwari S, Shukla VP, Biradar SR, Singh AK. Blur parameters identification for simultaneous defocus and motion blur. CSI Transactions on ICT:1-12.

[16] Candes EJ, Donoho DL. Curvelets. Manuscript. http://www.stat.stanford.edu/ $\sim$ donoho/Reports/1998/curvelets.zip. 1999.

[17] Candes EJ, Demanet L, Donoho DL, Ying L. Fast discrete curvelet transform. Technical report, CalTech. 2005.

[18] Candès E, Demanet L, Donoho DL, Ying L. CurveLab-2.1. 2.2008-08-15. http://www.curvelet.org. 2008.

[19] Vasumathi G, P Subashini. Pixel Classification of SAR ice images using ANFIS-PSO Classifier. Indonesian Journal of Electrical Engineering and Informatics (IJEEI) 4.4. 2016.

[20] Azriyenni A, Mustafa MW, Sukma DY, Dame ME. 2014. Backpropagation Neural Network Modeling for Fault Location in Transmission Line $150 \mathrm{kV}$. Indonesian Journal of Electrical Engineering and Informatics; 2(1): 1-12.

[21] Wachenfeld S, Terlunen S, Jiang X. 1-D barcode image database. http://cvpr.uni-muenster.de/ research/barcode. 2008.

[22] Szentandrási I, Dubská M, and Herout A. Fast detection and recognition of QR codes in highresolution images. Graph@FIT, Brno Institute of Technology. 2012. 